Check for updates

Cite this: RSC Adv., 2017, 7, 43114

\title{
Ethyl acetate extract of crabapple fruit is the cholesterol-lowering fraction
}

\author{
Dongshan Wang, $\uparrow$ Yingchun Wu, $\uparrow$ Chuhe Liu, Yahui Wang, Yin Li, Lingling Luo, \\ Yuanyuan Zhao, Zunli Ke, Cheng Huang (D* and Shengjie Fan*
}

Hypercholesterolemia is highly associated with cardiovascular diseases (CVDs) such as atherosclerosis. Our previous study showed that extracts of crabapple fruit may decrease serum total cholesterol (TC) and low-density lipoprotein cholesterol (LDL-C) in high-fat diet-induced obese mice. To investigate which bioactive component is responsive for cholesterol-lowering effect in crabapples and whether the component has effects on atherosclerosis, three different chemical fractions were isolated from ethanol extract of crabapples, and obese C57BL/6 mice fed with a high-fat diet and arteriosclerotic low-density lipoprotein receptor deficiency $\left(L D L R^{-1-}\right)$ mice fed with a western-diet (WD) were involved in our pharmacological studies. The results showed that ethyl acetate extract of crabapple (EAC) significantly decreased serum $T C$ in both DIO and $L D L R^{-/-}$mice. EAC also improved obesity, insulin resistance, lipid accumulation in the liver tissue in the DIO mice and prevented atherosclerotic plaque formation in $L D L R^{-/}$mice. Mechanistic study showed that EAC increased cholesterol excretion in the feces of DIO and $L D L R^{-/-}$mice, and elevated CYP7A1 mRNA in HepG2 cell and inhibited cholesterol accumulation in the cell membrane. Our data suggest that EAC may lower serum TC and block atherosclerotic plaque via the inhibition of intestinal cholesterol absorption and TC synthesis in the liver, and EAC may be used as a dietary supplement on hypercholesterolemia and atherosclerosis.

Received 13th June 2017

Accepted 28th August 2017

DOI: 10.1039/c7ra06585j

rsc.li/rsc-advances
For years, nutraceuticals offer a safe and cost-effective option for long-term treatment of hypercholesteremia. ${ }^{13}$ Crabapples, belonging to the genus of the family Rosaceae, are important ornamental plants widely distributed throughout the world. The crabapple fruits have been used as food and nutritional supply. Additionally, their fruits, flowers and roots are rich in polyphenols, flavonoids and dihydrochalcones which have managed in prevention and treatment of various diseases including digestive disease, cardiomyopathy, accelerated aging, tumor, and metabolism disorder. ${ }^{14-18}$ Recently, we have confirmed that the extracts of crabapple fruits could lower serum cholesterol and LDL-c in high-fat diet-induced C57BL/6 obese mice. ${ }^{19}$ However, the bioactive components for cholesterol lowering are unrevealed. Furthermore, whether the extracts can prevent atherosclerotic plaque formation is unknown.

In the present study, we refined several fractions, including ethyl acetate extract of crabapples (EAC). Pharmacological studies showed EAC decreased serum cholesterol in high-fat diet induced obesity mice and prevented atherosclerotic plaques formation in $L D L R^{-/-}$mice. Additionally, EAC regulated CYP7A1 mRNA expression in mice livers and cholesterol biosynthesis in HepG2 human liver cell line, indicating that ethyl acetate fraction may be the bioactive component and the cholesterol-lowering effect of EAC may achieve through CYP7A1 pathway.
School of Pharmacy, Shanghai University of Traditional Chinese Medicine, Shanghai 201203,China.E-mail: chuang@shutcm.edu.cn; Shengjiefan@shutcm.edu.cn

$\dagger$ Dongshan Wang and Yingchun Wu contributed equally to this work. 


\section{Materials and methods}

\section{Preparation and extractions}

Fresh crabapple fruits were obtained from Crabapple R\&D Company (Huailai, Hebei, China). The fruits were dried in electrothermal drying oven (DHG-9140A, China). Two kg of dried crabapples were extracted with $8 \mathrm{~L}$ ethanol by ultrasonic (KX, China) for 3 times each $1 \mathrm{~h}$. Then, the extract was concentrated at $40{ }^{\circ} \mathrm{C}$ with a rotary evaporator (EYELA N-1100SW, Japan) under reduced pressure. The extracts were dissolved in $2 \mathrm{~L}$ water and sequentially partitioned with chloromethane, ethyl acetate and $n$-butanol (Sinopharm Chemical Reagent Co., Ltd, Beijing, China) for three times, respectively. Finally, different fractions including chloromethane extract of crabapple (CC), ethyl acetate extract of crabapple (EAC) and $n$-butanol extract of crabapple (BC) were freeze-dried to powder by cryogenic dryer (Christ Alpha 1-2 LD plus, Germany) and stored at $-80{ }^{\circ} \mathrm{C}$ until use.

EAC was isolated through silica gel 60 GF254 (60-100 mesh, Merck) using chloromethane and methanol at ratio of $20: 1$, $15: 1,10: 1,5: 1$, and $1: 1$. Fractions were obtained from this separation (1-15) by analyzing of the thin-layer chromatography (TLC). Compounds were purified and isolated by with RP-18 reverse phase silica gel (YMC Co., Ltd, Tokyo), Sephadex LH20 column chromatography (GE Healthcare Bio-Sciences AB, USA) and semi-preparative HPLC (Agilent Technologies, Santa Clara, CA, USA).

The chemical construction of three compounds separating from EAC were identified by ${ }^{1} \mathrm{H}$ NMR and ${ }^{13} \mathrm{C}$ NMR spectroscopy analysis. Compound 1 was a white powder, ${ }^{1} \mathrm{H}$ NMR $(600 \mathrm{MHz}$, $\left.\mathrm{CDCl}_{3}\right)$ spectroscopic data showed $\delta 5.34(1 \mathrm{H}, \mathrm{brd}, J=5.1 \mathrm{~Hz}, \mathrm{H}-$ 6), 3.54 (1H, m, H-3), 1.01 (3H, s, H-19), 0.91 (3H, d, $J=6.4 \mathrm{~Hz}$, $\mathrm{H}-21), 0.85$ (3H, t, $J=7.5 \mathrm{~Hz}, \mathrm{H}-29), 0.84(3 \mathrm{H}, \mathrm{d}, J=6.8 \mathrm{~Hz}, \mathrm{H}-$ 26), $0.81(3 \mathrm{H}, \mathrm{d}, J=6.8 \mathrm{~Hz}, \mathrm{H}-27), 0.68$ (3H, s, H-18); ${ }^{13} \mathrm{C}$ NMR $\left(150 \mathrm{MHz}, \mathrm{CDCl}_{3}\right.$ ) spectroscopic data showed $\delta 141.0$ (C-5), 121.9 (C-6), 72.0 (C-3), 57.0 (C-14), 56.3 (C-17), 50.3 (C-9), 46.0 (C-24), 42.4 (C-13), 42.5 (C-4), 40.0 (C-12), 37.9 (C-1), 36.7 (C-10), 36.4 (C-20), 34.2 (C-22), 32.1 (C-7), 32.1 (C-8), 31.8 (C-2), 29.4 (C25), 28.5 (C-16), 26.3 (C-23), 24.5 (C-15), 23.4 (C-28), 21.4 (C-11), 20.1 (C-26), 19.6 (C-19), 19.3 (C-27), 19.0 (C-21), 12.2 (C-18), 12.1 (C-29). Compound 2 was a white amorphous powder, ${ }^{1} \mathrm{H}$ NMR $\left(600 \mathrm{MHz} \mathrm{CDCl}_{3}\right)$ spectroscopic data showed $\delta 5.48(1 \mathrm{H}, \mathrm{brs}, \mathrm{H}-$ 12), 3.59 (1H, d, $J=7.0 \mathrm{~Hz}, \mathrm{H}-3), 1.23$ (3H, d, $J=6.5 \mathrm{~Hz}, \mathrm{H}-29)$, 1.19 (3H, s, H-27), 1.04 (3H, s, H-25), 1.03 (3H, s, H-24), 1.03 (3H, s, H-26), 1.01 (3H, s, H-23), 0.88 (1H, d, $J=6.5 \mathrm{~Hz}, \mathrm{H}-30)$; ${ }^{13} \mathrm{C}$ NMR $\left(150 \mathrm{MHz}, \mathrm{CDCl}_{3}\right.$ ) spectroscopic data showed $\delta 179.7$ (C-28), 139.0 (C-13), 125.4 (C-12), 77.9 (C-3), 55.6 (C-5), 53.3 (C18), 47.8 (C-17), 47.8 (C-9), 42.3 (C-14), 39.7 (C-4), 39.3 (C-19), 39.1 (C-8), 39.1 (C-1), 38.8 (C-20), 37.2 (C-10), 37.0 (C-22), 33.3 (C-7), 30.8 (C-21), 28.5 (C-15), 28.4 (C-23), 27.8 (C-2), 24.6 (C-16), 23.6 (C-27), 23.4 (C-11), 21.1 (C-30), 18.5 (C-6), 17.3 (C-29), 17.2 (C-26), 16.9 (C-25), 15.4 (C-24). Compound 3 was a white amorphous powder, ${ }^{1} \mathrm{H}$ NMR (600 $\mathrm{MHz}, \mathrm{CD}_{3} \mathrm{OD}$ ) spectroscopic data showed $\delta 7.06\left(2 \mathrm{H}, \mathrm{d}, J=8.4 \mathrm{~Hz}, \mathrm{H}-2^{\prime}, 6^{\prime}\right), 6.68(2 \mathrm{H}, \mathrm{d}, J=$ $\left.8.4 \mathrm{~Hz}, \mathrm{H}-3^{\prime}\right), 6.15\left(1 \mathrm{H}, \mathrm{d}, J=1.4 \mathrm{~Hz}, \mathrm{H}-3^{\prime}\right), 5.95(1 \mathrm{H}, \mathrm{d}, J=$ $\left.1.4 \mathrm{~Hz}, \mathrm{H}-5^{\prime}\right), 5.86\left(2 \mathrm{H}\right.$, brs, H-6, 8), $5.03\left(1 \mathrm{H}, \mathrm{d}, J=6.8 \mathrm{~Hz}, \mathrm{H}-1^{\prime \prime}\right)$,
3.30-3.91 (6H, m, H-2"-6"), $3.45(1 \mathrm{H}, \mathrm{t}, J=8.0 \mathrm{~Hz}, \mathrm{H}-\alpha), 2.87$ $(2 \mathrm{H}, \mathrm{t}, J=8.0 \mathrm{~Hz}, \mathrm{H}-\beta) ;{ }^{13} \mathrm{C}$ NMR $\left(150 \mathrm{MHz}, \mathrm{CD}_{3} \mathrm{OD}\right)$ spectroscopic data showed $\delta 204.2$ (C-4), 164.6 (C-7), 164.2 (C-5, 9), 155.4 (C-4'), $131.6\left(\mathrm{C}-1^{\prime}\right), 129.1\left(\mathrm{C}-2^{\prime}, 6^{\prime}\right), 115.1\left(\mathrm{C}-3^{\prime}, 5^{\prime}\right), 103.7(\mathrm{C}-$ 10), 94.7 (C-6, 8), 102.1 (C-1"), 98.1 (C-7), 92.4 (C-5), $78.5\left(\mathrm{C}-5^{\prime \prime}\right)$, $77.7\left(\mathrm{C}-3^{\prime \prime}\right), 74.3\left(\mathrm{C}-2^{\prime \prime}\right), 71.2\left(\mathrm{C}-4^{\prime \prime}\right), 62.3\left(\mathrm{C}-6^{\prime \prime}\right), 45.4(\mathrm{C}-\alpha), 29.5$ $(\mathrm{C}-\beta)$.

\section{High-performance liquid chromatography (HPLC) analysis}

HPLC analysis was performed on an Agilent 1200 series HPLC system (Agilent Technologies, Santa Clara, CA, USA). Samples were dissolved in a mobile phase acetonitrile-water with the final concentration of $1 \%(1: 1, \mathrm{v} / \mathrm{v})$. After filtration by Millipore membrane $(0.45 \mu \mathrm{m})$ syringe filter, $10 \mu \mathrm{L}$ samples were taken by with a UV-DAD detector at a $\lambda$ max of $210 \mathrm{~nm}$. An autoinfection Agilent Extend-C18 of HPLC column $(5 \mu \mathrm{m}, 4.6 \times 250 \mathrm{~mm})$ was monitored to separate at $30{ }^{\circ} \mathrm{C}$. The samples were analyzed using a solvent system with $0.1 \%$ formic acid in water (A) and acetonitrile (B) under the following the method: 0-20 min, 5\% $\mathrm{B} ; 20.01 \mathrm{~min}, 85 \% \mathrm{~B} ; 30 \mathrm{~min}, 95 \% \mathrm{~B}$ at a flow rate of $1.0 \mathrm{~mL} \mathrm{~min}^{-1}$. Retention time of $\beta$-sitosterol, ursolic acid, phlorizin was compared to EAC.

\section{Total polyphenol and flavonoid content analysis in EAC}

The contents of total polyphenol and flavonoid in the EAC were tested by using methods of Folin-Ciocalteu and colorimetric respectively as previously reported. ${ }^{20}$ Results of total polyphenol and total flavonoids contents were presented as milligram of gallic acid of EAC or rutin per gram of EAC.

\section{Animal experiments}

Animal experiments were conducted in accordance with the guidelines and regulations of the Ethics Committee of Shanghai University of Traditional Chinese Medicine (Approved Number: 2015017). Male C57BL/6 mice were obtained from the SLAC Laboratory (Shanghai, China) at the six-week-old. The mice were housed under controlled temperature $\left(22-23{ }^{\circ} \mathrm{C}\right)$ with a $12 \mathrm{~h}$ light/dark cycle. After the adaptation period, the mice were fed with a high-fat $(\mathrm{HF})$ diet $(60 \%$ of calories derived from fat, $5.24 \mathrm{kcal} \mathrm{g}^{-1}$, Research Diets, D12492) for 3 months to induce obesity. ${ }^{21}$ For effective fraction screening, the diet-induced obese (DIO) mice were randomly grouped into $\mathrm{HF}$ diet or $\mathrm{HF}$ diet mixed with (CC, BC and EAC, $0.1 \%$, w/w) and an ethanol extract (EC) group $(1 \%, w / w)$ and treated for 2 weeks. For EAC study, the DIO mice were randomly grouped and treated with $0.015 \%$ atorvastatin (Tianfang Pharmaceuticals, China) as a positive control, or different concentrations of EAC $(0.1 \%$, $0.2 \%, 0.5 \%)$ or HF diet continuously along with chow diet $(10 \%$ of calories derived from fat, Research Diets no. D12450B) as a lean control for 2 weeks. All diet and water were provided freely. Body weight and food intake were recorded every other day.

The $L D L R^{-/-}$mice were purchased from Animal Center, Nanjing University (Nanjing, China). Five-week-old male $L D L R^{-/-}$ mice were randomly divided and fed with a Western diet (HC, $0.21 \%$ cholesterol, $41 \%$ of calories derived from fat, $4.7 \mathrm{kcal} \mathrm{g}^{-1}$, 
Table 1 Sequence of the primers used in real-time PCR

\begin{tabular}{|c|c|c|}
\hline Gene & Forward primer & Reverse primer \\
\hline$\beta$-Actin & TGTCCACCTTCCAGCAGATGT & AGCTCAGTAACAGTCCGCCTAGA \\
\hline$L X R \alpha$ & GAGTGTCGACTTCGCAAATGC & CCTCTTCTTGCCGCTTCAGT \\
\hline$A P O E$ & GAACCGCTTCGGGTTGATTCCAAA & TCAGTGCCGTCAGTTCTTGTG \\
\hline$L D L R$ & CTCCACTCTA TGGGATTACCT & ACTGAAAATGGCTTCGTTTATGAC \\
\hline SREBP-2 & ACAACACTGACCAGCACCCATAC & AAGACGCTCAAGACAATCACACC \\
\hline
\end{tabular}

Research Diets no. D12079B) or a Western diet mixed with atorvastatin $(0.015 \%)$ or EAC $(0.1 \%, 0.2 \%$, and $0.5 \%)$ along with chow diet for 14 weeks. ${ }^{22}$ Body weight and food intake were measured every other day.

\section{Intraperitoneal glucose tolerance test (IPGTT)}

At the end of treatments, the DIO and $L D L R^{-/-}$mice were fasted overnight. The glucose levels were tested from the tail vein ( $0 \mathrm{~min})$. Then, glucose ( $1 \mathrm{~g}$ per $\mathrm{kg}$ body weight) was intraperitoneally injected and additional blood glucose levels were measured at regular intervals (15, 30, 60, 90 and $120 \mathrm{~min})$.

\section{Serum biochemistry analysis}

At the end of the experiments, the heart blood samples were collected after anesthesia (urethane, $20 \%$, w/v) ${ }^{23}$ Blood samples were centrifuged at $3000 \mathrm{rpm}$ for $15 \mathrm{~min}$ to separate serum. Serum triglyceride (TG), TC, high-density lipoprotein cholesterol (HDL-c) and LDL-c, alanine aminotransferase (ALT), aspartate transaminase (AST) levels were measured by using an automatic analyzer (Hitachi 7020, Tokyo, Japan) with $150 \mu \mathrm{L}$ of heart blood serum.

\section{Hematoxylin and eosin (H\&E) staining}

For analysis the morphology of liver tissue, the liver samples were fixed in $4 \%$ paraformaldehyde, embedded in paraffin and sliced into $10 \mu \mathrm{m}$ sections according to a standard procedure. The sections were stained with H\&E. Images were then examined under a microscope (Zeiss, Germany).

\section{Liver and fecal lipid content analysis}

The liver tissues $(50 \mathrm{mg}$ ) were weighed and suspended with $0.5 \mathrm{~mL}$ lysis buffer (20 mM Tris-HCl, pH 7.5, $150 \mathrm{mM} \mathrm{NaCl}$, and $1 \%$ Triton) and an equal volume of chloroform to test the lipid concentration of liver. The chloroform layer was separated, dried, and finally dissolved in $100 \mu \mathrm{L}$ of isopropyl alcohol to measure the TG and TC levels as described. ${ }^{24}$ The mouse feces were collected over the final 3 days and freeze-dried and then grinded. Fecal lipids was extracted and measured using the same method as liver concentrations.

\section{Cell culture}

HepG2 cells on the cover slide were grown in DMEM without serum for $24 \mathrm{~h}$. Then the cells were transferred to DMEM containing $10 \%$ fetal bovine serum (Hyclone, Logan, UT, USA), and treated with EAC, $\beta$-sitosterol, ursolic acid, phlorizin, or pravastatin at the indicated concentrations. After $24 \mathrm{~h}$ treatment, the cell fixed in 4\% paraformaldehyde for $10 \mathrm{~min}$, and incubated with $100 \mu \mathrm{L}$ of cholesteryl BODIPY (Life Technologies, Sigma, USA) for $30 \mathrm{~min}$ at $37{ }^{\circ} \mathrm{C}$. Then the cell was counterstained with $3.2 \mu \mathrm{L}$ of Fluoroshield DAPI (Life Science, Sigma, USA). Pictures were taken using a fluorescence microscope (Zeiss, Germany).

\section{Quantitative real-time polymerase chain reaction (qRT-PCR)}

qRT-PCR was performed as previous described. ${ }^{20}$ Briefly, total RNA from DIO mouse livers were isolated using the TRIzol (Takara, Tokyo, Japan) according to the protocol of manufacturer. ${ }^{25}$ The first-strand cDNA was synthesized with a RevertAid First Strand cDNA synthesis kit (Thermo Scientific, Wilmington, Delaware, USA). The gene expression levels were analyzed by the quantitative real-time PCR using an ABI StepOnePlus Real-Time PCR system (Applied Biosystems, CA, USA). The sequences of the primers (Generay Biotech Co., Ltd, Shanghai, China) used in the experiment were shown in Table 1 . The mRNA levels were normalized using $\beta$-actin as an internal reference.

\section{Analysis of atherosclerotic plaque}

The anatomy of aortic vessels were stained with Sudan IV for $15 \mathrm{~min}$, then destained $10 \mathrm{~min}$ using $80 \%$ ethanol to quantify atherosclerosis of the aortic arch, which is easy to develop atherosclerotic plaque. Sudanophilic lesions were assessed by microscope (Lecia, German) computer-assisted image analysis and calculated the area of plaque.

\section{Statistical analysis}

The results are expressed as mean \pm SEM. Data analyses were performed using SPSS 21.0 for windows statistical program. Statistical analysis was programmed by one-way analysis of variance (ANOVA). Difference was regarded as significant $\left({ }^{*} P<\right.$ $0.05)$.

\section{Results}

\section{EAC is cholesterol-lowering faction in crabapples}

Our pervious study showed that ethanol extract of crabapple fruits had anti-hypercholesteremia effect. ${ }^{19}$ To identify the bioactive fraction for the treatment of hyperlipidemia, the crabapple extract was refined with chloromethane, $n$-butanol and 

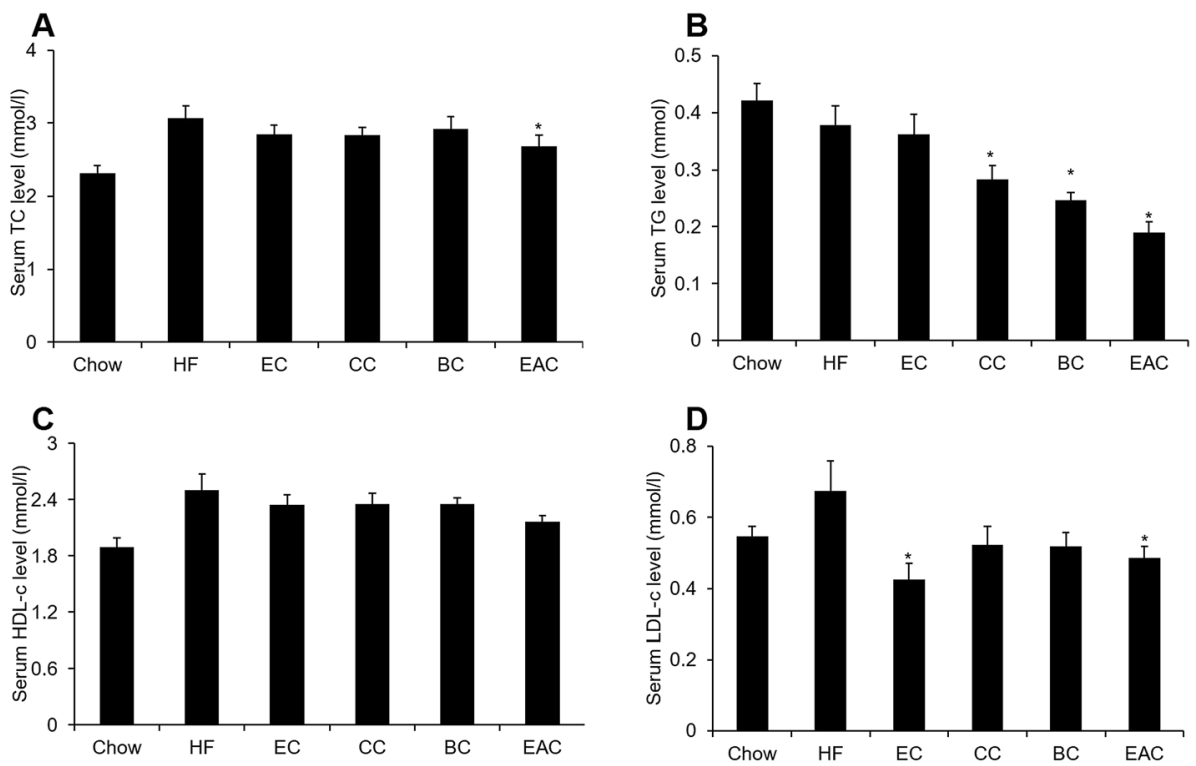

Fig. 1 EAC has anti-hypercholesteremia effect. (A-D) Different extractions of EC, CC, BC, EAC treated with DIO mice of serum total cholesterol (TC), triglyceride (TG), and high-density lipoprotein cholesterol (HDL-C) levels, low-density lipoprotein cholesterol (LDL-c). Data are presented as mean \pm SEM for eight mice per group. $* P<0.05$ vs. HF group.

ethyl acetate to obtain three fractions: chloromethane extract (CC), $n$-butanol extract (BC) and ethyl acetate extract (EAC).

To assay the cholesterol-lowering bioactive component of crabapples, male C57BL/6 mice were fed with high-fat diet (HF) to induce obesity (DIO) for 3 months. The crabapple fractions $\mathrm{CC}, \mathrm{BC}$ and EAC were mixed into high-fat diet at $0.1 \%, \mathrm{w} / \mathrm{w}$. An ethanol extract mixed at $1 \%, \mathrm{w} / \mathrm{w}$ was used for control. After 2 weeks of the treatment on the DIO mice, $0.1 \%$ of EAC reduced serum TC, TG and LDL-c remarkably, while EAC did not change HDL-c (Fig. 1A-D), suggesting EAC may have effect on hyperlipidemia than other 2 fractions.

\section{Chemical composition analysis of EAC}

Based on the result above, the EAC seemed the bioactive component of crabapples. To investigate the main components of EAC, we measured total polyphenol and flavonoid with FolinCiocalteu and colorimetric assay. The data showed that total polyphenol content was $614.90 \pm 15.20 \mathrm{mg}$ gallic acid equivalents per $\mathrm{g}$ EAC, total flavonoid was $206.82 \pm 8.61 \mathrm{mg}$ rutin equivalents per $\mathrm{g}$ EAC, indicating that EAC were rich in flavonoids and polyphenols, which similar to the previous studies. $^{26-28}$

We then analyzed the major three compounds purified from crabapples. Contrasted the NMR spectrum data with literature, ${ }^{29-31}$ the compound $1,2,3$ were identified $\beta$-sitosterol, ursolic acid and phlorizin (Fig. $2 \mathrm{~A}-\mathrm{C}$ ). Then we analyzed EAC components by High Performance Liquid Chromatography (HPLC) with a UV-DAD detector at a $\lambda$ max of $210 \mathrm{~nm}$. Under this condition, only 3 chromatographic peaks were determined (Fig. 2D), except for the solvent peak. Comparing with the retention times of standard controls, we identified the 3 peaks were same as $\beta$-sitosterol, ursolic acid and phlorizin (Fig. 2B-D), suggesting that the major contents in EAC are $\beta$-sitosterol, ursolic acid and phlorizin.

\section{EAC improves metabolic disorders in DIO mice}

To test the effect of EAC on metabolic disorders, the DIO mice were randomly divided into 5 groups and treated with different dosages of EAC $(0.1 \%, 0.2 \%, 0.5 \%, \mathrm{w} / \mathrm{w})$ mixed into a HF diet or with atorvastatin $(0.015 \%, \mathrm{w} / \mathrm{w})$ as a positive control or fed with a high-fat diet alone. After 2 week treatment, high-fat diet increased body weight while atorvastatin increased slimly (Fig. 3A and B). However, EAC decreased body weight dosedependently without change of food intake (Fig. 3A-C), indicating body weight loss of EAC was not relevant with food intake.

Blood glucose homeostasis is closely related to lipid metabolism. ${ }^{32,33}$ To investigate EAC effect on glucose and lipid balance, fasting blood glucose and short-term effects of EAC on the blood glucose levels of the DIO mice were tested. As expected, high-fat diet induced higher fasting blood glucose levels and impaired glucose tolerance, compared to the chow control mice (Fig. 3D-F). Atorvastatin and three different concentration of EAC decreased fasting blood glucose in DIO mice (Fig. 3D). EAC, especially $0.1 \%$ and $0.5 \%$ of EAC, also reduced blood glucose at different time point ( $30 \mathrm{~min}, 60 \mathrm{~min}, 120 \mathrm{~min}$ ) after i.p. injection of glucose comparing with HF in IPGTT (Fig. 3E). The total area under the curve (AUC) of blood glucose levels between 0 and 120 min showed $0.1 \%, 0.5 \%$ of EAC and atorvastatin were significantly lowered than $\mathrm{HF}$ group while there was no change on $0.2 \%$ of EAC (Fig. 3F). The results indicated that EAC could decrease the fasting blood glucose and improve the glucose tolerance in DIO mice.

Importantly, serum TG, TC, LDL-c and HDL-c levels were increased significantly in HF group comparing with chow 

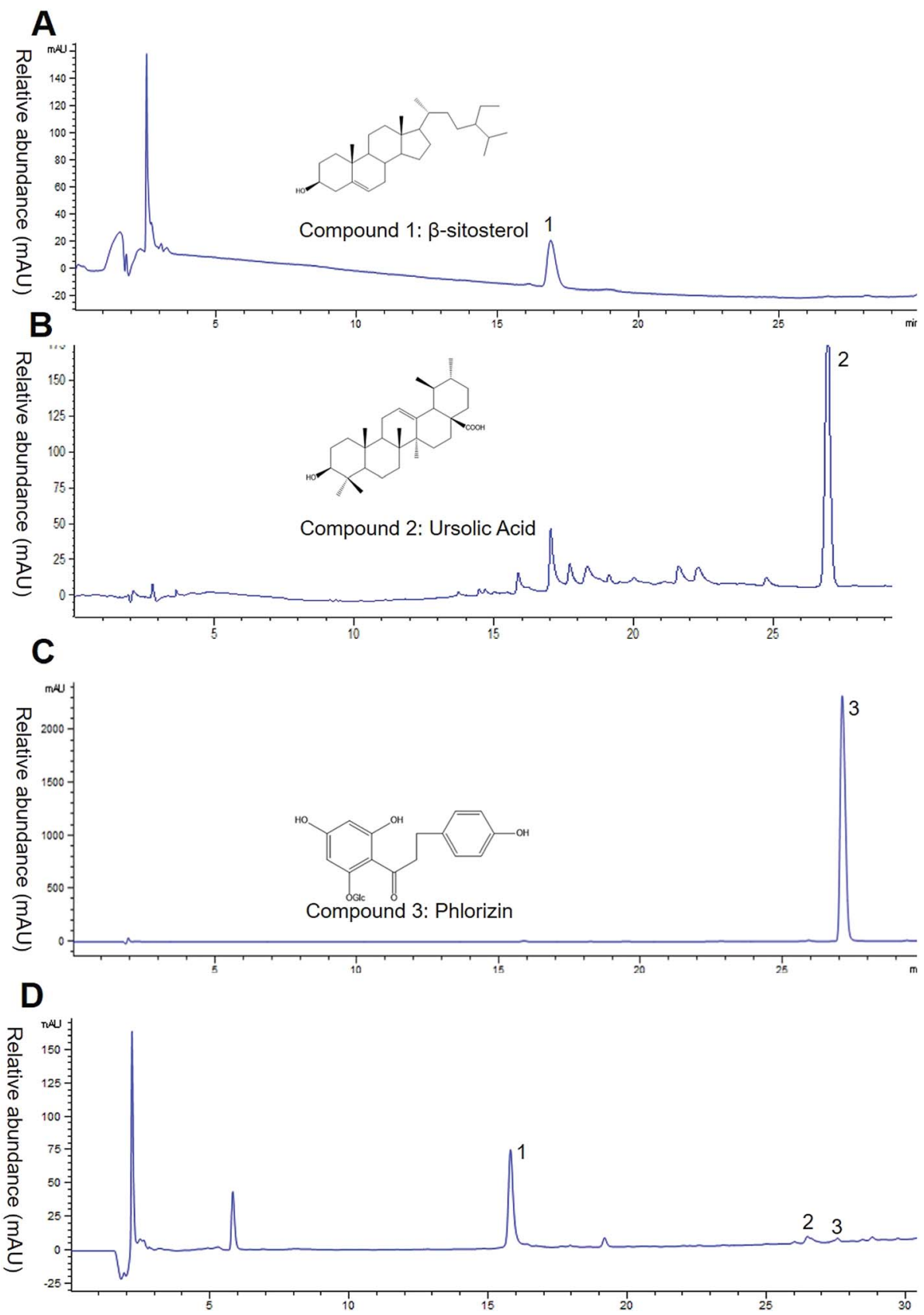

Fig. 2 Fingerprint analysis of EAC by RP-HPLC. (A-C) $\beta$-Sitosterol, ursolic acid, phlorizin were analyzed as standards using HPLC with a C-18 reverse phase column at $210 \mathrm{~nm}$ and chemical construction. (D) The major components of the EAC were detected for comparison with the retention times of the standard compounds.

group. However, $0.2 \%$ and $0.5 \%$ of EAC reduced TC and LDL-C markedly with a dose-dependent manner (Fig. 3G). Moreover, high concentration of EAC decreased HDL-c. Interestingly, the positive control, $0.015 \%$ of atorvastatin reduced LDL-c, but not TC in DIO mice (Fig. 3G), indicating that $0.2 \%$ and $0.5 \%$ of EAC had anti-hyperlipidemia effect, especially on cholesterol.

Serum ALT and AST are commonly biomarkers for identification of liver health. ${ }^{34}$ Fig. $3 \mathrm{H}$ showed $\mathrm{HF}$ increased obviously indicating that high-fat diet can induce liver tissue damage. However, $0.2 \%$ and $0.5 \%$ of EAC as well as atorvastatin decreased significantly serum ALT levels dose-dependently (Fig. $3 \mathrm{H}$ ), but not the serum AST (Fig. 3I), indicating that EAC could improve liver damage.

\section{EAC ameliorates hepatic steatosis in DIO mice}

Liver is an important organ for lipid metabolism. Obese mice usually develop fatty liver, even hepatic steatosis, due to excess storage of fat in liver. ${ }^{35}$ To test whether EAC may alleviate fatty liver, we performed $\mathrm{H} \& \mathrm{E}$ staining to observe liver structure of the mice. As expected, HF group showed 


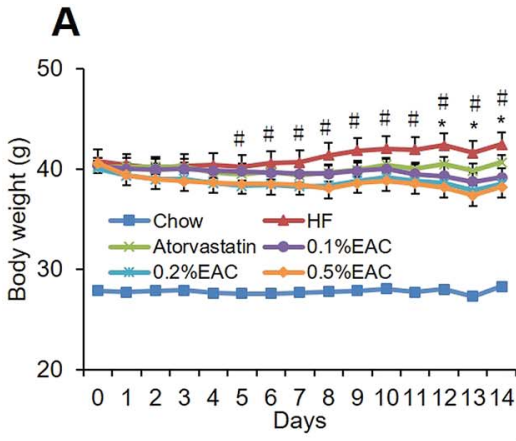

B
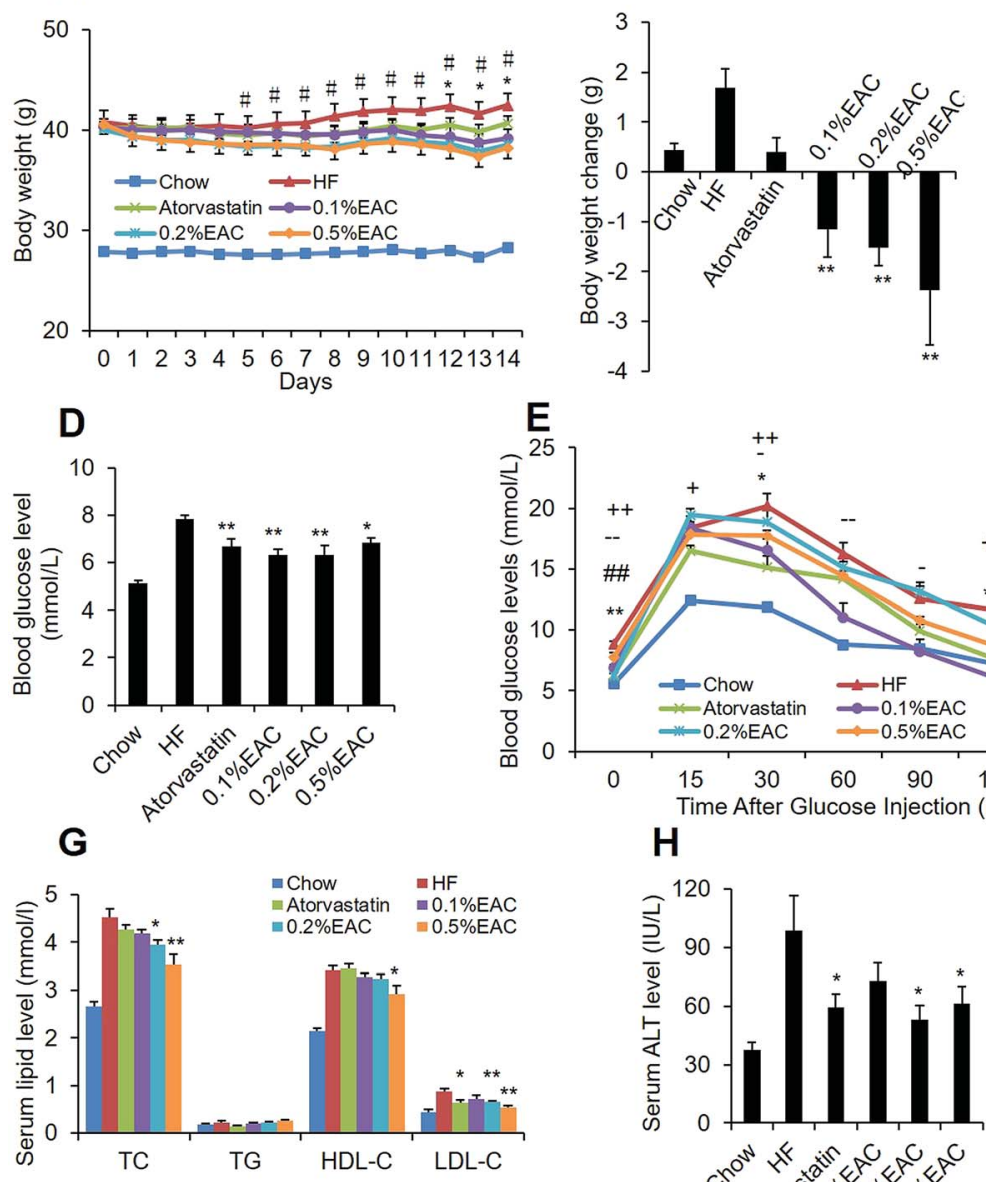

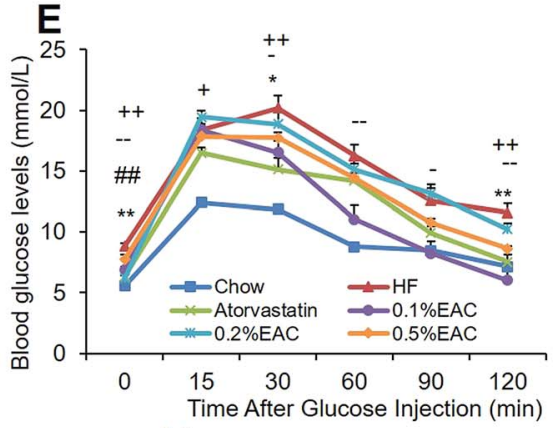

$\mathrm{H}$

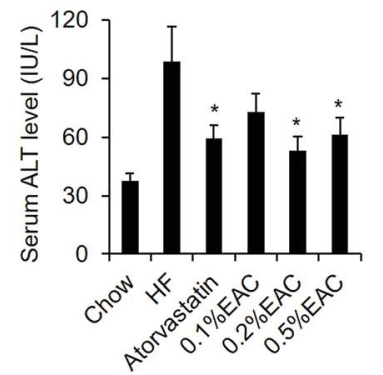

C
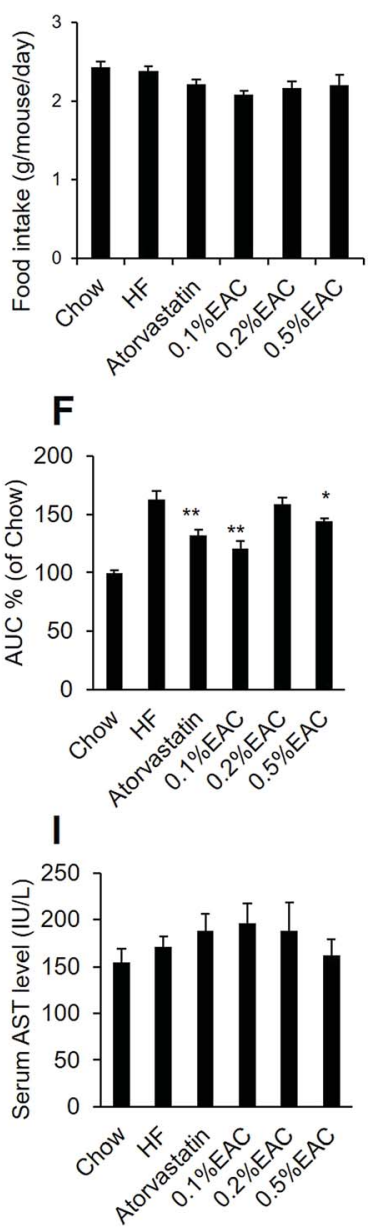

Fig. 3 EAC improves metabolic disorders in DIO mice. (A \& B) Body weight change; (C) food intake amount; (D) fasting blood glucose; (E) intraperitoneal glucose tolerance test (IPGTT); (F) quantification of the area under the curve (AUC) from the GTT in (E). (G) Serum total cholesterol (TC), triglyceride (TG), low-density lipoprotein cholesterol (LDL-C), and high-density lipoprotein cholesterol (HDL-C) levels; (H \& I) serum ALT, AST level. Data are presented as mean \pm SEM for eight mice per group. (A \& E) ${ }^{++} P<0.01,{ }^{+} P<0.05$, atorvastatin vs. $\mathrm{HF} ;{ }^{--} P<0.01,-P<0.05,0.1 \%$ EAC vs. HF; ${ }^{\# \#} P<0.01,{ }^{\#} P<0.05,0.2 \%$ EAC vs. HF; $* * P<0.01, * P<0.05,0.5 \%$ EAC vs. HF; $(\mathrm{B}, \mathrm{D}, \mathrm{F}, \mathrm{G}$ and H): $* * P<0.01, * P<0.05$ vs. HF group.

obviously lipid droplets in liver compared to Chow group (Fig. 4A). However, different concentrations of EAC-treated and atorvastatin group showed much less lipid droplets comparing with HF group with a dose-dependent manner (Fig. 4A atorvastatin, 0.1\% EAC, 0.2\% EAC and 0.5\% EAC), while lipid accumulation in atorvastatin seemed more effective than high concentration of EAC.

As known, accumulation of triglycerides (TG) in hepatocytes is the main cause of fatty liver formation. ${ }^{36}$ Thus, we measured TG and TC level in liver tissues (Fig. 4B and C). Comparing with Chow group, TG content in HF group was highly increased. In accordance with $\mathrm{HE}$ staining, $0.2 \%$ and $0.5 \%$ of EAC lowered TG dose-dependently, while no change in atorvastatin (Fig. 4B). However, there was no difference among the 6 groups in TC content in liver (Fig. 4C). These data indicate that EAC prevents liver damage and fatty liver formation in DIO mice.

Furthermore, we measured cholesterol and triglyceride contents in mouse feces. Fig. 4D showed that AC increased TC contents in feces with a severe dose-dependent pattern when compared with HF group, suggesting that EAC inhibited the absorption of TC in the intestine. However, EAC treatment had no effect on TG in feces (Fig. 4E).

Taken together, EAC decreased serum TC and LDL-c levels, inhibited lipid accumulation in liver and TC absorption in the intestine in DIO mice.

EAC prevents the hypercholesterolemia in the $L D L R^{-/-}$mice

To further confirm the anti-hypercholesterolemia effect of EAC, LDL receptor-knockout $\left(L D L R^{-/-}\right)$mice were involved in our study, which is a proper model for hypercholesterolemia and atherosclerosis. ${ }^{37,38} L D L R^{-/-}$mice were fed with Western diet with high-cholesterol for 14 weeks to induce hypercholesterolemia and atherosclerotic plaque. Meanwhile, the mice were treated with different dosage of EAC $(0.1 \%, 0.2 \%$, and $0.5 \%)$ or atorvastatin $(0.015 \%)$ as a positive control with chow diet as the regular control (Chow). After 14 week treatment, EAC had no 


\section{A}
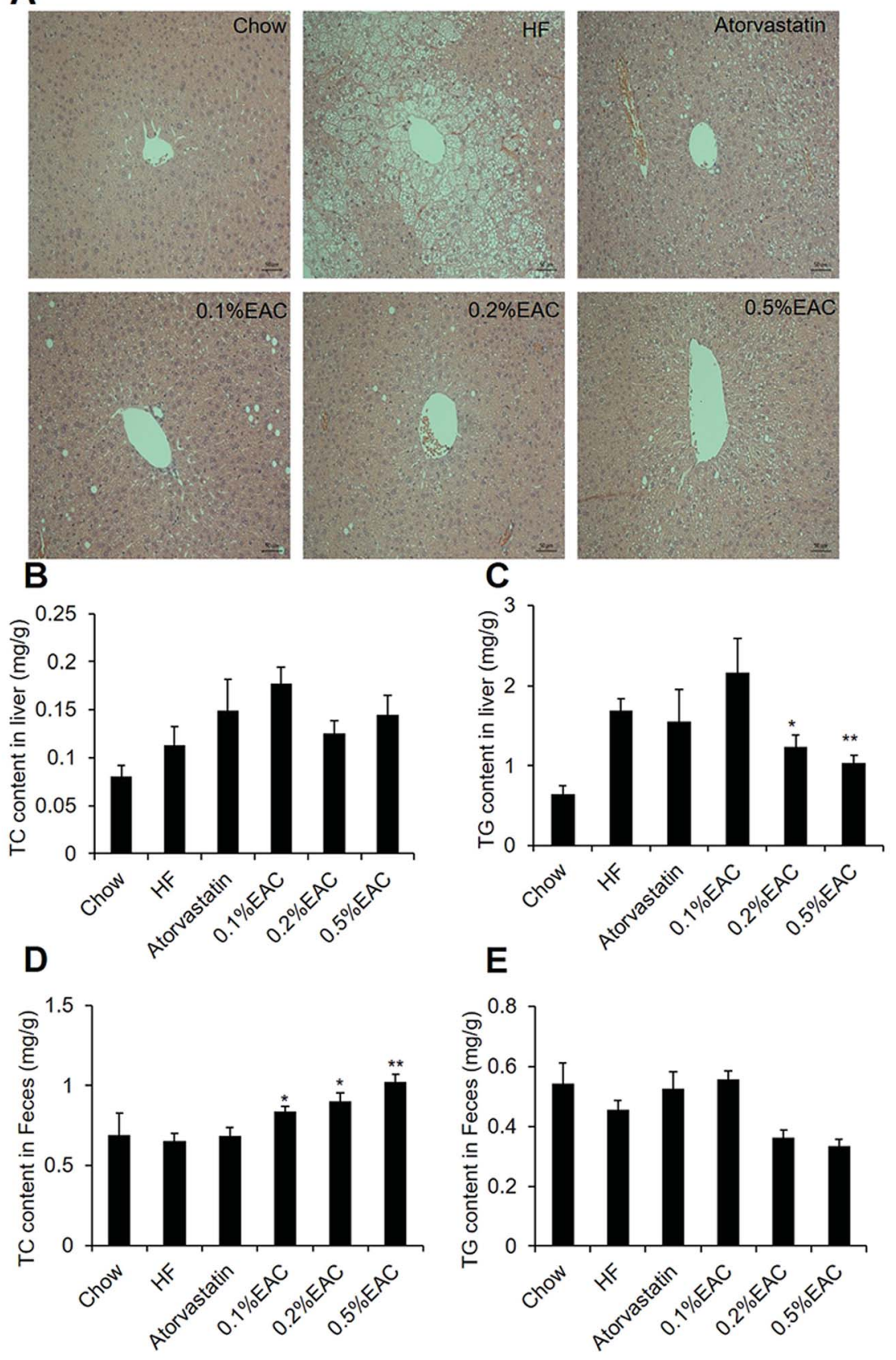

Fig. 4 EAC ameliorates the hepatic steatosis in DIO mice. (A) H\&E staining of liver sections ( $\times 200)$; (B) liver cholesterol level; (C) liver triglyceride level; (D) fecal cholesterol level; (E) fecal triglyceride level. Data are presented as mean $\pm \mathrm{SEM}$ for seven mice per group. $* * P<0.01, * P<0.05$ vs. HF group.

effect on body weight, food intake or fasting blood glucose in $L D L R^{-1-}$ mice (Fig. 5A-C). However, 3 different concentrations of EAC decreased serum TC and TG levels comparing with HC group in $L D L R^{-1-}$ mice, while atorvastatin decreased TC, but not TG (Fig. 5D). And $0.2 \%$ of EAC showed also decreased HDL-c and LDL-c as well as atorvastatin (Fig. 5D). These data confirmed that EAC has anti-hypercholesterolemia effect in vivo.

Additionally, the aortic arch were peeled off and stained with Sudan IV to observe the aortic plaque formation (Fig. 5G). Comparing with Chow, $\mathrm{HC}$ appeared obvious red aortic plaques and the biggest aortic plaque area (Fig. $5 \mathrm{H}$ ) among the 6 groups.
However, the aortic plaque area was much less in atorvastatin and EAC groups (Fig. 5G and $\mathrm{H}$ ), suggesting that EAC can inhibit aortic plaque formation in $L D L R^{-/-}$mice induced by $\mathrm{HC}$ diet.

We also measured serum ALT and AST (Fig. 5E and F), TC and TG in the liver tissues (Fig. 5I and J). However, the data showed that EAC could not change any of them. Interestingly, in agreement with the DIO data, EAC increased TC in $L D L R^{-/-}$ mouse feces dose-dependently (Fig. 5K) but not TG in feces (Fig. 5L), which indicated that EAC could inhibit TC absorption in intestines. 
A
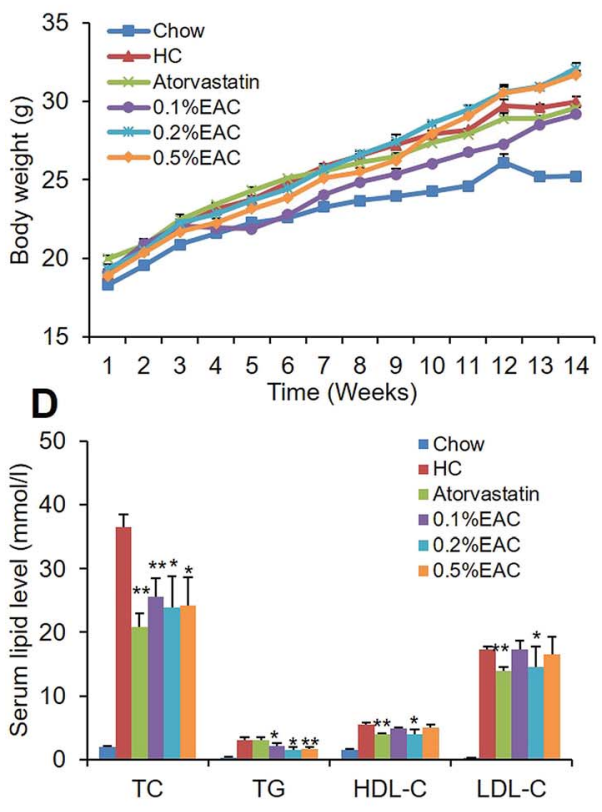

G
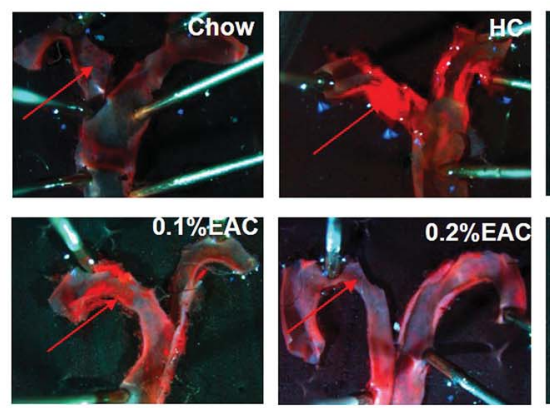

I

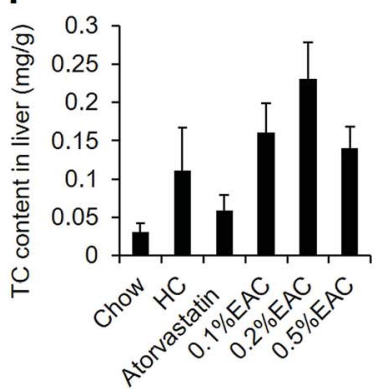

B
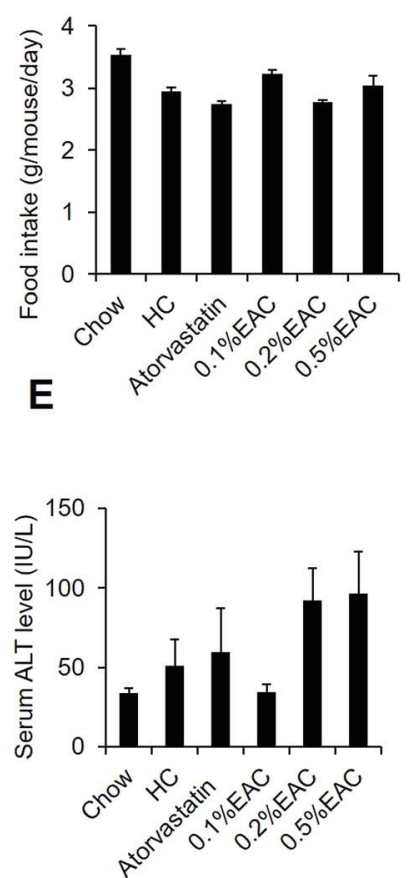

C
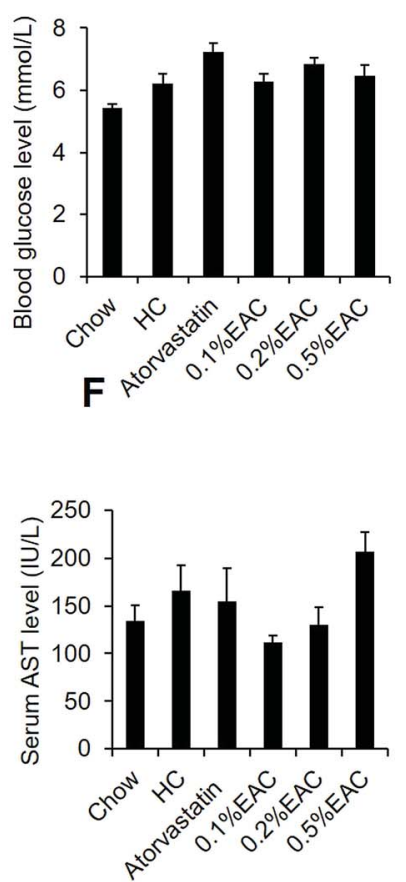

H
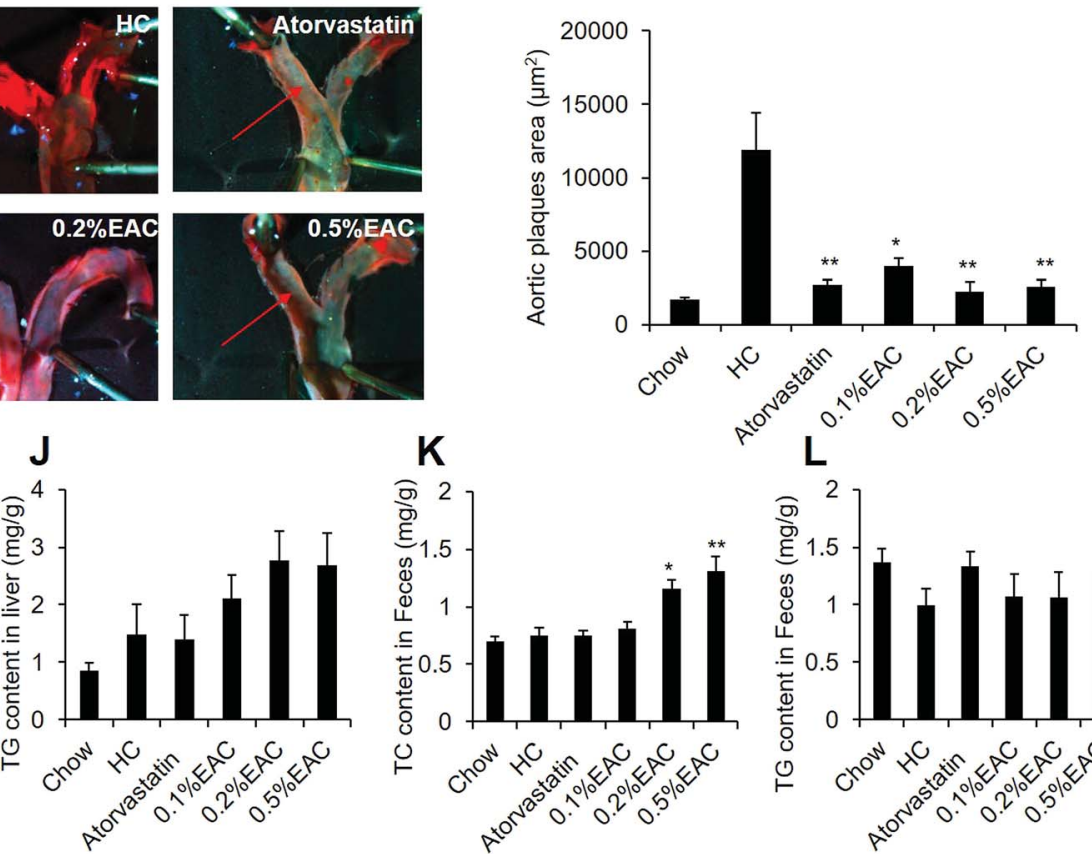

$\mathrm{K}$

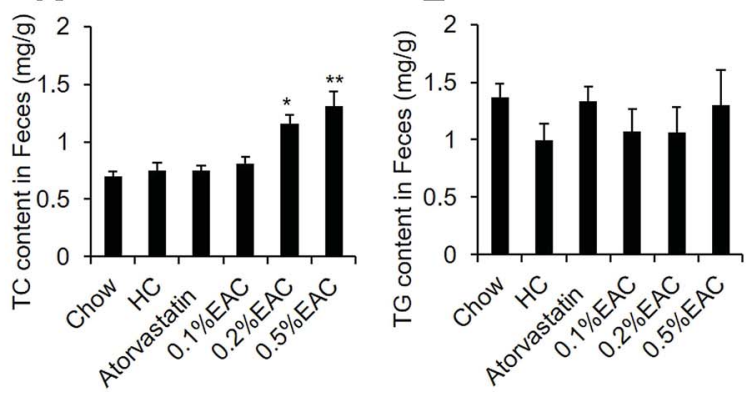

Fig. 5 EAC decreases the atherosclerosis development in the $L D L R^{-/-}$mice. (A) Body weight change; (B) food intake amount; (C) fasting blood glucose level; (D) serum TC, TG, HDL-C, LDL-c levels; (E) serum ALT level; (F) serum AST level; (G) representative photographs of aortas arch lesion; (H) quantitation of aortas lesion area; (I) liver cholesterol level; (J) liver triglyceride level; (K) fecal cholesterol level; (L) fecal triglyceride level. Data are presented as mean \pm SEM for seven mice per group. $* * P<0.01, * P<0.05$ vs. HC group.

EAC and its small molecules inhibit the cholesterol biosynthesis in HepG2 cells

As known, cholesterol-lowering strategies are mainly through reducing the absorption, promoting excretion, and decreasing the synthesis of cholesterol. ${ }^{39}$ Our results had proved that EAC increased fecal TC to promote cholesterol excretion. Whether EAC could affect cholesterol biosynthesis was unknown. To further investigate the mechanism of EAC effect on cholesterollowering, a BODIPY-cholesterol assay was used for visualizing membrane cholesterol accumulation in HepG2 cells. 
A
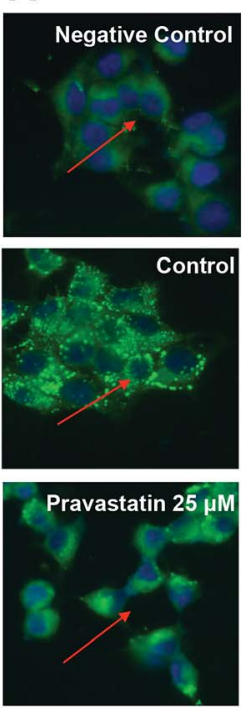

B

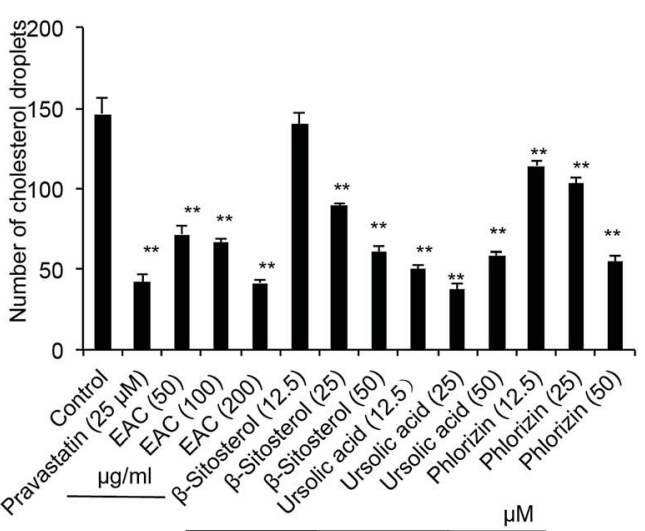

$\beta$-Sitosterol
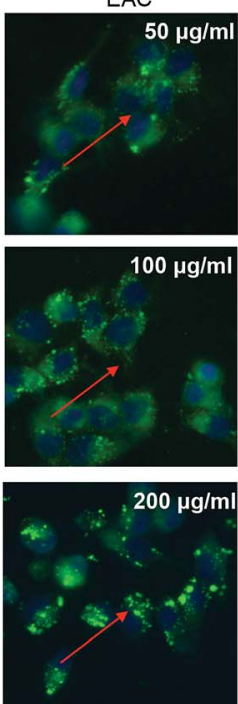

*
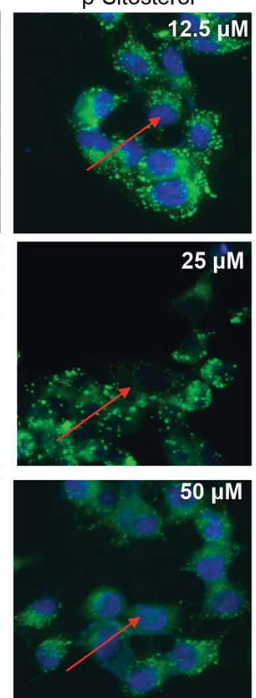

c
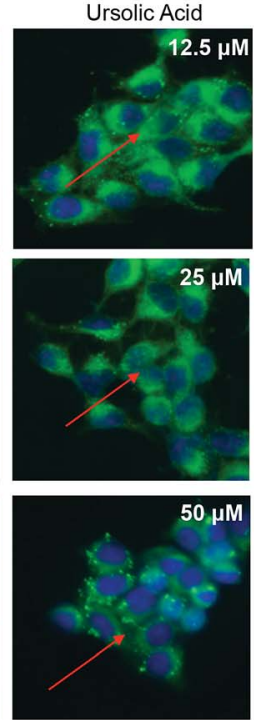

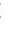
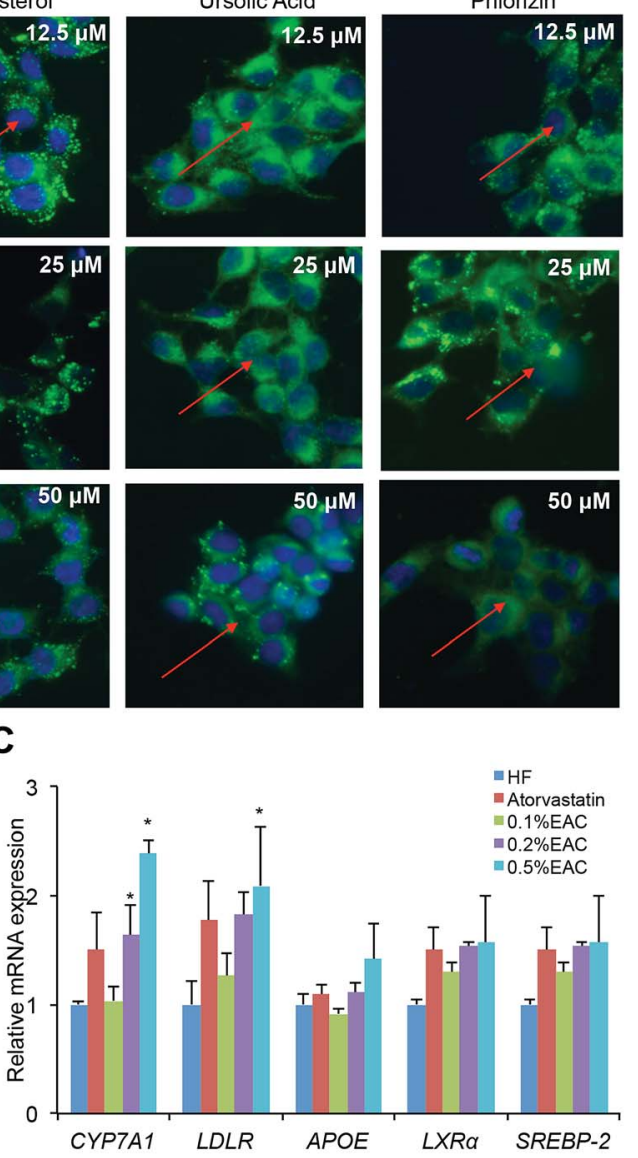

$0 \mu \mathrm{M}$ 
potential mechanism of anti-hypercholesterolemia remains further studies. In this study, we used the EAC from crabapples and confirmed that EAC lowered serum cholesterol dosedependently in DIO mice. Additional, EAC lowered serum TC and blocked atherosclerotic plaque formation in $L D L R^{-/-}$mice. Further, the mechanism study showed that EAC increased CYP7A1 mRNA level in DIO mouse liver tissue. We identified that $\beta$-sitosterol, ursolic acid and phlorizin are three major components in EAC and the compounds inhibited cholesterol biosynthesis in vitro. This result is agreement to the previous reports that show $\beta$-sitosterol, ursolic acid and phlorizin have an effect on lipid metabolism. ${ }^{40-42}$ Thus, it is possible that $\beta$ sitosterol, ursolic acid and phlorizin responsible for the cholesterol-lowering effects of crabapple.

High-fat diet induced obesity (DIO) mice is one of authority animal model to mimic metabolic syndrome with obesity, diabetes, dyslipidemia and fatty liver. ${ }^{43}$ Although EAC decreased body weight gain in DIO mice without changing energy input, and decreased fasting blood glucose and improved glucose tolerance (Fig. 3), the reason is unclear. If this beneficial effect on obesity and diabetes relays on EAC antihypercholesterolemia effect or some other mechanism required further study. It may also be a concern that EAC inhibited lipid accumulation in liver (Fig. 4A-C) without changing liver TC level. One possibility is that the main component in liver lipid droplets is TG. Although EAC did not change TC content in liver, TG decreased by EAC treatment to improve fatty liver induced by high-fat diet. And both pharmacological study and mechanism study showed that the effective dose of EAC is $0.2 \%$ and $0.5 \%$, not $0.1 \%$.

Interestingly, in both DIO and $L D L R^{-/-}$mice, EAC increased fecal TC significantly and with an obvious dose-dependent manner. It indicated that EAC may inhibit TC absorption to reduce serum TC. $\beta$-Sitosterol is structurally similar to cholesterol and may compete with it in the intestine to inhibit absorption of dietary cholesterol. ${ }^{44,45}$ Therefore, $\beta$-sitosterol may be the active element in crabapples for the inhibition of TC absorption.

Another possible mechanism for the TC lowering effects is the EAC increased CYP7A1 expression in the mice. CYP7A1 is an attractive target for cholesterol lowering drug development sense CYP7A1 may increase biotransformation from cholesterol to bile acid in liver. The findings are similar to the results in our previous study that showed 5 different species of crabapple increase CYP7A1 mRNA and protein levels in DIO mouse liver. The data indicated that EAC may inhibit TC synthesis in the liver through the increase of CYP7A1 function.

\section{Conclusion}

We identified that crabapple fraction EAC has significant cholesterol-lowering effect in both DIO and $L D L R^{-1-}$ mice. Additionally, EAC may improve obesity, insulin resistance, lipid accumulation in the liver tissue and may prevent atherosclerotic plaque formation in $L D L R^{-/-}$mice. In vitro study showed that EAC inhibited cholesterol accumulation in HepG2 cell membrane. Mechanistic study found that EAC inhibited TC absorption in the intestine and elevated mRNA of CYP7A1 in the liver of the mice. Our data suggests that EAC is a potential functional dietary supplement on hypercholesterolemia and atherosclerosis.

\section{Conflicts of interest}

The authors declare no conflict of interest.

\section{Acknowledgements}

This work was supported by National Natural Science Foundation of China (81703774) and Sailing Plan (16YF1411600) to S. F., and Modernization of Chinese Materia Medica Project (15401902300) to C. H. from Shanghai Science and Technology Committee.

\section{References}

1 U. E. Bauer, P. A. Briss, R. A. Goodman and B. A. Bowman, Lancet, 2014, 384, 45-52.

2 M. K. Ito, Ann. Pharmacother., 2012, 46, 1368-1381.

3 N. J. Stone, J. G. Robinson, A. H. Lichtenstein, C. N. B. Merz, C. B. Blum, R. H. Eckel, A. C. Goldberg, D. Gordon, D. Levy and D. M. Lloydjones, J. Am. Coll. Cardiol., 2014, 63, 28892934.

4 L. D. Colantonio, V. Bittner, K. Reynolds, E. B. Levitan, R. S. Rosenson, M. Banach, S. T. Kent, S. F. Derose, H. Zhou and M. M. Safford, Circulation, 2016, 133, 256.

5 S. Mitchell, S. Roso, M. Samuel and M. Pladevall-Vila, $B M C$ Cardiovasc. Disord., 2016, 16, 74.

6 R. H. Nelson, Prim. Care Clin. Office Pract., 2013, 40, 195-211.

7 L. I. Xue-Jun and B. Yan, Int. J. Intern. Med., 2009, 96, 106108.

8 S. G. Mlodinow, M. K. Onysko, J. W. Vandiver, M. L. Hunter and T. D. Mahvan, Clinician Rev., 2014, 24, 41.

9 H. Sinzinger, R. Wolfram and B. A. Peskar, J. Cardiovasc. Pharmacol., 2002, 40, 163-171.

10 S. Ramkumar and A. Wilson, Heart, Lung Circ., 2015, 24, S190.

11 M. D. Müller-Wieland and N. Marx, Herz/Kreislauf, 2016, 41, 1-6.

12 J. R. Anderson, Drug Therapeut. Bull., 2016, 54, 18.

13 Z. Y. Chen, R. Jiao and K. Y. Ma, J. Agric. Food Chem., 2008, 56, 8761-8773.

14 F. Aladedunye and B. Matthäus, Food Chem., 2014, 159, 273281.

15 N. P. Seeram, R. H. Cichewicz, A. Chandra and M. G. Nair, J. Agric. Food Chem., 2003, 51, 1948-1951.

16 S. Q. Wang, X. F. Zhu, X. N. Wang, T. Shen, F. Xiang and H. X. Lou, Phytochemistry, 2013, 87, 119-125.

17 X. Wei, R. Zhao, Y. H. Sun, J. P. Cong, F. G. Meng and H. M. Zhou, J. Enzyme Inhib. Med. Chem., 2009, 24, 234-240.

18 Q. Xiaoxiao, X. Yun Feng, Z. Zhiqin and Y. Yuncong, Molecules, 2015, 20, 21193-21203. 
19 M. Li, S. Xue, S. Tan, X. Qin, M. Gu, D. Wang, Y. Zhang, L. Guo, F. Huang and Y. Yao, J. Funct. Foods, 2016, 27, 416-428.

20 L. Yu, W. Cai, Y. Zhang, L. Feng and C. Huang, J. Funct. Foods, 2015, 14, 278-288.

21 Q. Yang, M. Qi, R. Tong, D. Wang, L. Ding, Z. Li, C. Huang, Z. Wang and L. Yang, Int. J. Mol. Sci., 2017, 18, 1393.

22 Y. Zhang, X. Wang, C. Vales, F. Y. Lee, H. Lee, A. J. Lusis and P. A. Edwards, Arterioscler., Thromb., Vasc. Biol., 2006, 26, 2316-2321.

23 K. Inai, K. Arihiro, Y. Takeshima, S. Yonehara, Y. Tachiyama, N. Khatun and T. Nishisaka, Cancer Sci., 1991, 82, 380-385.

24 J. J. Tang, J. G. Li, W. Qi, W. W. Qiu, P. S. Li, B. L. Li and B. L. Song, Cell Metab., 2011, 13, 44-56.

25 A. B. Hummon, S. R. Lim, M. J. Difilippantonio and T. Ried, BioTechniques, 2007, 42, 467-470.

26 E. G. Rudikovskaya, L. V. Dudareva, A. A. Shishparenok, N. B. Mitanova, I. G. Petrova and A. V. Rudikovskii, Chem. Nat. Compd., 2014, 50, 640-641.

27 Y. Luo, C. Zhang, C. Xu, S. Zuo and Y. Liu, Asia-Pacific Traditional Medicine, 2014, 10, 20-22.

28 N. Li, J. Shi and K. Wang, J. Agric. Food Chem., 2014, 62, 574581.

29 P. Zhuang, W. Fu and C. Tan, Res. Dev. Nat. Prod., 2009, 21, 963-965.

30 X. Y. Yu, Chin. Tradit. Herb. Drugs, 2011, 42, 661-663.

31 G. E. Yong-Bin, J. Chin. Med. Mater., 2014, 37, 435-438.
32 G. Hao, D. Wang, Y. Sun, J. Yu, F. Lin and H. Cao, Biomed. Rep., 2017, 6, 339-345.

33 R. Akcılar, F. E. Koçak, H. Şimșek, A. Akcılar, Z. Bayat, E. Ece and H. Kökdaşgil, Iran. J. Basic Med. Sci., 2016, 19, 245-251.

34 G. F. Webster, T. G. Webster and L. R. Grimes, Dermatol. Online J., 2016, 23, 34929.

35 M. Jakobsen, T. Berentzen, T. Sørensen and K. Overvad, Epidemiol. Rev., 2007, 29, 77-87.

36 A. Yoo, V. P. Narayan, E. Y. Hong, W. K. Whang and T. Park, Sci. Rep., 2017, 7, 2251.

37 K. Y. Park, E. Oh, M. K. Kwak, H. S. Jun and T. H. Heo, PLoS One, 2016, 11, e0150791.

38 M. Shibata, E. Shibata, K. Maemura, Y. Kondo and M. Harada-Shiba, Med. Mol. Morphol., 2017, 50, 130-144.

39 Y. Chang and J. Robidoux, Curr. Opin. Pharmacol., 2017, 33, 47-55.

40 Y. Jia, M. J. H. Bhuiyan, H. J. Jun, H. L. Ji, M. H. Hoang, H. J. Lee, N. Kim, D. Lee, K. Y. Hwang and Y. H. Bang, Bioorg. Med. Chem. Lett., 2011, 21, 5876-5880.

41 J. Köhler, D. Teupser, A. Elsässer and O. Weingärtner, Br. J. Pharmacol., 2017, 11, 1281-1289.

42 T. T. Shen, S. W. Liu, J. Zhao, M. C. Wang, Z. Y. Chang and H. Wang, Food Sci., 2014, 35, 192-195.

43 M. B. Schulze and F. B. Hu, Diabetes Care, 2004, 27, 613-614. 44 M. L. Fernandez and S. Vega-López, Cardiovasc. Drug Rev., 2005, 23, 57-70.

45 K. Lu, M. H. Lee and S. B. Patel, Trends Endocrinol. Metab., 2001, 12, 314-320. 